\title{
Integrative research agenda for diagnosis in sepsis
}

\author{
Katie Nolan ${ }^{1}$, Ruth O'Leary ${ }^{1}$, Lieuwe D. J. Bos ${ }^{2}$, Ignacio Martin-Loeches ${ }^{1}$ \\ ${ }^{1}$ Multidisciplinary Intensive Care Research Organization (MICRO), St. James's Hospital Dublin, Trinity Centre for Health Sciences, Trinity College (TCD), \\ Irish Centre for Vascular Biology (ICVB), Dublin, Ireland; ${ }^{2}$ Department of Intensive Care, Academic Medical Center, Amsterdam, the Netherlands \\ Correspondence to: Ignacio Martin-Loeches. Department of Intensive Care Medicine, St. James's Hospital, James's St, Ushers, Dublin 8, D08 NHY1, \\ Ireland. Email: drmartinloeches@gmail.com. \\ Comment on: Morley D, Torres A, Cillóniz C, et al. Predictors of treatment failure and clinical stability in patients with community acquired \\ pneumonia. Ann Transl Med 2017;5:443.
}

Submitted Jun 03, 2017. Accepted for publication Jun 09, 2017.

doi: $10.21037 / \mathrm{atm} .2017 .06 .57$

View this article at: http://dx.doi.org/10.21037/atm.2017.06.57

Intensive care medicine deals with the most critically and time-sensitive patients in the hospital. Each patient requires individualised point-of-care (POC) management and continuous care. In recent years high rates of sepsis and septic shock in intensive care unit (ICU) medicine have become omni-present. Sepsis is a severe systemic inflammatory response in which the host response to infection causes organ dysfunction and raises mortality risk. The time-sensitive nature of sepsis is exacerbated by the variable nature of presentation and a lack of medical consensus on diagnostic parameters. Correct diagnosis and treatment must take into account variables such as the infectious agent, the primary site of infection, patient comorbidities as well as the individual host response (1).

\section{Sepsis in the ICU}

Epidemiologic data on sepsis mortality in the ICU varies considerably between countries. The 2014 Intensive Care over Nations (ICON) audit identified approximately 10,000 ICU patients with an ICU mortality rate of $16.2 \%$ compared to $25.8 \%$ in ICU patients with sepsis (2). In hospitals, community acquired pneumonia (CAP) is the most common cause of sepsis with ventilator associated pneumonia (VAP) due to nosocomial infection more common in the ICU setting (3). Rapid recognition and diagnosis of respiratory infection and sepsis is crucial to appropriate management of patients.

Previously sepsis was diagnosed using systemic inflammatory response syndrome (SIRS) criteria. Two or more SIRS criteria with the presence of infection indicate sepsis. Sepsis criteria, organ dysfunction and hypotension indicate severe sepsis. Severe sepsis criteria and hypotension despite adequate fluid resuscitation indicates septic shock. SIRS criteria are temperature $>38$ or $<36$, heart rate: $90 / \mathrm{min}$, respiratory rate: $20 / \mathrm{min}$ or white cell count: 12,000 or $4000 / \mathrm{mm}^{3}$ (4). While SIRS criteria is still utilised to diagnose sepsis, it is under heavy review and revision. The highly variable nature of sepsis symptoms means that some patients present without $2+$ SIRS criteria and the validity of SIRs diagnosis remain unclear (5).

International revision of sepsis criteria produced Sepsis-3 criteria. Analysis found the sequential organ failure assessment (SOFA) to have greater prognostic accuracy than SIRS criteria but was not suitable for early risk identification. A SOFA score $>2$ indicates a mortality risk of $10 \%$ in hospitalised patients with suspected sepsis. A quick SOFA score was also developed as bedside criteria to assess patients for poor outcomes $(6,7)$.

Due to symptom heterogeneity and time urgency, definitive sets of protocols called "care bundles" are routinely used to streamline diagnosis and improve patient outcomes (8). The Surviving Sepsis Campaign and Sepsis-3 care bundles define sets of approved protocols for treatment of suspected sepsis and help streamline the response to a patient presenting with suspected sepsis. While care bundles remove some heterogeneity to patient care, a definitive diagnosis and tailored treatment would decrease unnecessary antibiotic use and improve patient outcomes (9).

\section{Novel diagnostics}

Owing to the time-pressures surrounding sepsis diagnosis 


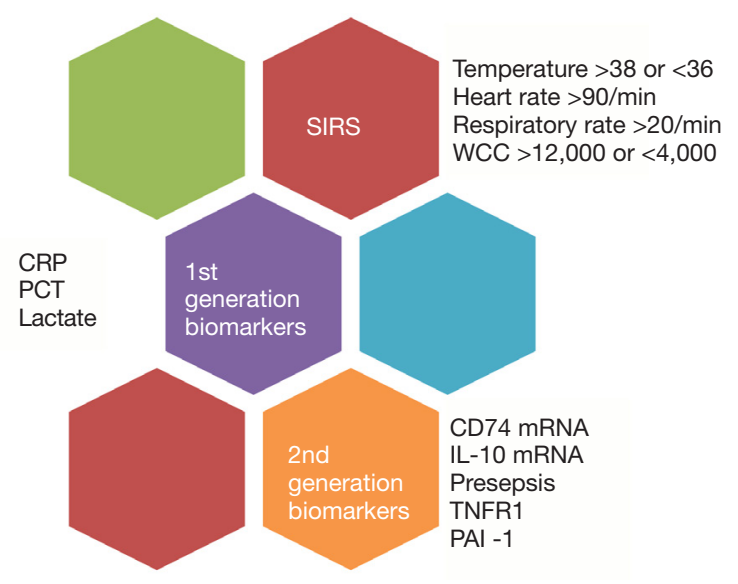

Figure 1 Evolution of diagnostic markers for sepsis. From SIRS to next generation biomarkers. SIRS, systemic inflammatory response syndrome; CRP, C-reactive protein; PCT, procalcitonin; TNFR1, tumour necrosis factor receptor 1 ; PAI-1, plasminogen activator inhibitor 1 .

and treatment, utilising novel technologies for rapid bedside diagnostics could greatly improve response times and sepsis treatment in the ICU. Advances in genomics and transcriptomics have highlighted the possibility of novel biomarker development for identifying sepsis (10). Recent sepsis diagnostics highlight a role for non-specific biomarkers of infection such as C-reactive protein (CRP) and procalcitonin (PCT). PCT has been indicated as a promising early biomarker of sepsis and PCT guided intervention has demonstrated a decrease in antibiotic duration in critically ill patients, an important consideration for antibiotic stewardship (11). Investigations are on-going for biochip immunoassay platforms for biomarkers such as CRP and PCT (12). Blood lactate levels are another nonspecific sepsis marker and research suggests that a POC lactate device may highlight patients at risk of sepsis and requiring resuscitation $(13,14)$.

While PCT, CRP and lactate are broad biomarkers of sepsis, novel sepsis—specific biomarkers are important for targeted therapy and to inform clinical decision-making. Urinary antigen testing for legionella and streptococcus pneumoniae demonstrated rapid, accurate and noninvasive testing methods. The use of quantitative-real time polymerase chain reaction might impact the determination of immunoparalysis in critically ill patients with one study reporting a decrease in CD74 mRNA and high levels of IL-10 mRNA expression being associated with a higher risk of nosocomial infections in ICU (15). Another novel marker, Presepsin, a soluble CD14 subtype has also been identified as an early diagnostic indicator of sepsis as well as being implicated in assessment of sepsis severity and prognosis (16). Tumour necrosis factor receptor 1 (TNFR1) and plasminogen activator inhibitor 1 (PAI-1) are both novel biomarkers for VAP which is a major risk factor for development of sepsis in the ICU (17).

The development and establishment of novel genomic biomarkers in daily clinical practise requires both a diagnostic platform and rigorously controlled testing (Figure 1). POC testing at the patient bedside would afford clinicians the speed required for treating sepsis. Sample turnaround time needs to be rapid. Currently blood culture testing and nucleic acid testing are used to identify bloodstream infections and isolate pathogens (18). Immediate future research is becoming more focused on microfluidic lab-on-a-chip (LOC) devices which can rapidly analyse blood samples from patients with suspected sepsis (19). While research to refine microfluidic chip detection of biomarkers in patient samples is ongoing, there is promising evidence that a disposable POC device for rapid, high sensitivity detection of sepsis in ICU patients is coming down the pipeline $(20,21)$.

\section{Defining patients for inclusion}

The problems with defining sepsis criteria and uniformly treating patients mean physicians and researchers are looking to randomised control trails (RCTs) for novel therapies. The use of RCTs in heterogeneous sepsis patient populations poses its own set of problems. Patients are acutely unwell and highly variable which can damage study sample sizes and poses problems for study design. In the first instance, patients enrolled in RCTs need to be uniformly representative to allow for correct interpretation of data. It is very important to differentiate between patients with sepsis and septic shock if accurate treatment analysis is to be used. Furthermore, use of a suitable control group is crucial for result analysis and study integrity however suitable control interventions and investigator "blinding" make defining control group criteria difficult. Defining strict inclusion criteria for sepsis RCTs may overcome some obstacles posed by patient heterogenicity but the trial may then be faced with slow recruitment and smaller sample sizes yielding low specificity results (22). This idea of precision medicine versus a population-based approach in ICU medicine is key. It is hoped that with the Sepsis-3 criteria and qSOFA 
criteria in sepsis diagnosis, defining patients that subscribe to a particular clinical phenotype could be achieved using novel molecular diagnostic platforms and could further advance diagnosis and treatment (23).

\section{Conclusions}

Sepsis and septic shock raise mortality rates for patients in the ICU. Multiple pathogens and variable symptom presentation make diagnosis and rapid treatment very difficult. Misdiagnosis leads to excessive antibiotic use, haemodynamic compromise and high mortality risk. New technologies focused on bench-to-bedside translational medicine could enable handheld sample analysis in minutes. Novel POC diagnostics could revolutionise sepsis management and mortality risk. As ICU medicine evolves, new diagnostic technologies will ensure rapid, accurate sepsis identification and help direct patient management and reduce mortality.

\section{Acknowledgements}

None.

\section{Footnote}

Conflicts of Interest: The authors have no conflicts of interest to declare.

\section{References}

1. van der Poll T. Future of sepsis therapies. Crit Care 2016;20:106.

2. Vincent JL, Marshall JC, Namendys-Silva SA, et al. Assessment of the worldwide burden of critical illness: the intensive care over nations (ICON) audit. Lancet Respir Med 2014;2:380-6.

3. Martin-Loeches I, Garnacho-Montero J, Nseir S. Focus on infection and sepsis 2017. Intensive Care Med 2017.

4. Singer M, Deutschman CS, Seymour CW, et al. The Third International Consensus Definitions for Sepsis and Septic Shock (Sepsis-3). JAMA 2016;315:801-10.

5. Kaukonen KM, Bailey M, Pilcher D, et al. Systemic inflammatory response syndrome criteria in defining severe sepsis. N Engl J Med 2015;372:1629-38.

6. Freund Y, Lemachatti N, Krastinova E, et al. Prognostic Accuracy of Sepsis-3 Criteria for In-Hospital Mortality Among Patients With Suspected Infection Presenting to the Emergency Department. JAMA 2017;317:301-8.

7. Giamarellos-Bourboulis EJ, Tsaganos T, Tsangaris I, et al. Validation of the new Sepsis-3 definitions: proposal for improvement in early risk identification. Clin Microbiol Infect 2017;23:104-9.

8. Barochia AV, Cui X, Vitberg D, et al. Bundled care for septic shock: an analysis of clinical trials. Crit Care Med 2010;38:668-78.

9. Dellinger RP, Levy MM, Rhodes A, et al. Surviving Sepsis Campaign: international guidelines for management of severe sepsis and septic shock, 2012. Intensive Care Med 2013;39:165-228.

10. Skibsted S, Bhasin MK, Aird WC, et al. Bench-to-bedside review: future novel diagnostics for sepsis - a systems biology approach. Crit Care 2013;17:231.

11. Wacker C, Prkno A, Brunkhorst FM, et al. Procalcitonin as a diagnostic marker for sepsis: a systematic review and meta-analysis. Lancet Infect Dis 2013;13:426-35.

12. Kemmler M, Sauer U, Schleicher E, et al. Biochip pointof-care device for sepsis diagnostics. Sensors and Actuators B: Chemical 2014;192:205-15.

13. Gaieski DF, Drumheller BC, Goyal M, et al. Accuracy of Handheld Point-of-Care Fingertip Lactate Measurement in the Emergency Department. West J Emerg Med 2013;14:58-62.

14. Ismail F, Mackay WG, Kerry A, et al. The accuracy and timeliness of a Point Of Care lactate measurement in patients with Sepsis. Scand J Trauma Resusc Emerg Med 2015;23:68.

15. Peronnet E, Venet F, Maucort-Boulch D, et al. Association between mRNA expression of CD74 and IL10 and risk of ICU-acquired infections. A multicenter cohort study. Intensive Care Medicine 2017. [Epub ahead of print].

16. Zhang J, Hu ZD, Song J, et al. Diagnostic Value of Presepsin for Sepsis: A Systematic Review and MetaAnalysis. Medicine (Baltimore) 2015;94:e2158.

17. Martin-Loeches I, Bos LD, Povoa P, et al. Tumor necrosis factor receptor 1 (TNFRI) for ventilator-associated pneumonia diagnosis by cytokine multiplex analysis. Intensive Care Med Exp 2015;3:26.

18. Afshari A, Schrenzel J, Ieven $M$, et al. Bench-to-bedside review: Rapid molecular diagnostics for bloodstream infection--a new frontier? Crit Care 2012;16:222.

19. Yang J, Selvaganapathy PR, Gould TJ, et al. A microfluidic device for rapid quantification of cell-free DNA in patients with severe sepsis. Lab Chip 2015;15:3925-33.

20. Zirath H, Peham J, Schnetz G, et al. A disposable 
microfluidic chip for rapid and sensitive detection of plasma biomarkers. Procedia Engineering 2014;87:496-9.

21. Sanjay ST, Fu G, Dou M, et al. Biomarker detection for disease diagnosis using cost-effective microfluidic platforms. Analyst 2015;140:7062-81.

Cite this article as: Nolan K, O’Leary R, Bos LD, MartinLoeches I. Integrative research agenda for diagnosis in sepsis. Ann Transl Med 2017;5(22):454. doi: 10.21037/atm.2017.06.57
22. Kampmeier TG, Ertmer C, Rehberg S. Translational research in sepsis-an ultimate challenge? Exp Transl Stroke Med 2011;3:14.

23. Martin-Loeches I, Bos L, Cobb JP. ARDS and precision medicine. ICU managment \& practise 2017;17:16-7. 